\title{
Fatores sociodemográficos moderam a associação da prática de atividade física dos pais e amigos com o nível de atividade física dos adolescentes?
}

http://dx.doi.org/10.11606/1807-5509202000040577

\author{
Gerfeson Mendonça */** \\ J acqueline de Oliveira Mendes* \\ Luanna Alexandra Cheng* \\ J oana Marcela Sales de Lucena* \\ J osé Cazuza de Farias J únior ${ }^{* * * *}$
}

*Universidade de Pernambuco, Recife, PE, Brasil.

**Universidade Federal

da Paraíba, João

Pessoa, PB, Brasil.

\begin{abstract}
0 objetivo deste estudo foi analisar se os fatores sociodemográficos moderam a associação da prática de atividade física dos pais e dos amigos com o nível de atividade física dos adolescentes. Trata-se de um estudo epidemiológico transversal, realizado com 2.859 adolescentes (57,8\% sexo feminino) de 14 a 19 anos de idade do município de João Pessoa (PB). 0 nível de atividade física dos adolescentes e a prática de atividade fisica dos pais e dos amigos foram mensurados por questionários. A prevalência de adolescentes fisicamente ativos foi de 50,2\% (IC95\%: 47,3-53,1), sendo mais elevada no sexo masculino (66,3\%; IC95\%: 63,7-69,0) comparada ao feminino (38,4\%; IC95\%: 36,1-40,8). Os resultados da análise multivariável demostraram que há associação entre a prática de atividade física dos pais e dos amigos e o nível de atividade física dos adolescentes. No entanto, essa associação é moderada por fatores sociodemográficos (sexo e classe econômica). A magnitude da medida de associação entre a prática de atividade física dos amigos e a dos adolescentes foi mais elevada nos adolescentes do sexo masculino e de classe econômica alta. A prática de atividade física da mãe se associou de forma significativa e positiva com o nivel de atividade física nos adolescentes de classe econômica alta. Conclui-se que os fatores sociodemográficos moderam a associação da prática de atividade física dos amigos e da mãe com o nivel de atividade física dos adolescentes. A magnitude da associação entre essas variáveis variou com o sexo e a classe econômica dos adolescentes.
\end{abstract}

Palavras-Chave: Atividade motora; Adolescência; Pais; Amigos.

\section{Introdução}

A atividade física na adolescência tem sido alvo de diversas investigaçóes ${ }^{1,2}$, sobretudo as que buscam entender qual o motivo de alguns adolescentes conseguirem ser fisicamente ativos e outros não ${ }^{3,4}$. A influência social está entre os fatores correlatos mais investigados e que tem se associado à atividade física dos adolescentes ${ }^{3-5}$. Essa influência pode se dá pelo fornecimento de apoio social, normas e modelação do comportamento em relação à prática de atividade física ${ }^{5-7}$.

A modelação do comportamento para a prática de atividade física por parte dos pais e dos amigos representa uma das formas de influência social mais investigada ${ }^{5,8,9}$. Tem sido caracterizada como uma aprendizagem observacional, no qual o comportamento de pessoas socialmente importantes passa a ser adotado pelos adolescentes como um modelo de comportamento ${ }^{8}$. Nesse sentido, a prática de atividade física dos pais e dos amigos pode representar um modelo para a prática dos adolescentes ${ }^{10}$.

Os resultados dos estudos que analisaram as relaçôes entre a prática de atividade física dos pais e a atividade física dos filhos (adolescentes) demonstraram que pais fisicamente ativos são mais propensos a terem filhos igualmente ativos fisicamente ${ }^{11,12}$.

$\mathrm{O}$ mesmo tem sido identificado em relaçáo 
à prática de atividade física dos amigos e a dos adolescentes ${ }^{13}$, apesar do número de estudos ainda ser relativamente pequeno comparado aos dos pais $^{14,15}$. Entretanto, os resultados destes estudos são conflitantes para confirmar as hipóteses de que essas associaçóes variam conforme características sociodemográficas dos adolescentes como sexo, idade e classe econômica dos pais e dos amigos.

Os estudos têm demonstrado que os adolescentes do sexo feminino, os mais velhos e os que pertencem aos estratos socioeconômicos mais baixos são menos ativos fisicamente ${ }^{1,2}$. Desse modo, é possível que a influência da modelação dos hábitos de atividade física dos pais e dos amigos sobre a atividade física dos filhos (amigos) seja diferente entre esses subgrupos, indicando que os fatores sociodemográficos atuariam como moderadores dessa associação. Uma variável moderadora é aquela que modifica o sentido ou a magnitude da medida de associação entre uma variável independente e a dependente, sendo essa associação diferente para uma ou mais das categorias dessa variável (moderadora) ${ }^{16}$.

Em estudo de revisão sistemática SeAbra et al. ${ }^{15}$ identificaram que não é consensual o efeito moderador do sexo em relaçáo a influência da prática de atividade física do pai e/ou da mãe sobre o nível de atividade física dos filhos e filhas,

\section{Método}

Estudo epidemiológico transversal, de base escolar, baseado na análise dos dados de um estudo mais amplo intitulado Nivel de atividade fisica e fatores associados em adolescentes do ensino médio na cidade de João Pessoa, PB: uma abordagem ecológica, com escolares de ambos os sexos, de 14 a 19 anos de idade, do ensino médio das redes pública e privada no município de João Pessoa (PB), realizada em 2009.

Para determinar o tamanho da amostra, os seguintes parâmetros foram adotados: prevalência estimada de 50\% (300 minutos ou mais por semana de atividade física); erro máximo aceitável de três pontos percentuais; intervalo de confiança de 95\%; efeito de desenho (deff) igual a dois e acréscimo de $30 \%$ para perdas e recusas, resultando em uma amostra estimada em 2.686 adolescentes.

A seleção da amostra foi por conglomerados em dois estágios (seleção sistemática de 30 escolas, distribuídas proporcionalmente por tipo respectivamente. Em meta-analise, YAO e RHODES ${ }^{17}$ mostram que há resultados de estudos que apontam associaçôes significativas entre a atividade física da mãe e das filhas e entre a do pai e a dos filhos e outros resultados identificaram associaçóes significativas entre a atividade física do pai e ambos os filhos ou da mãe e ambos os filhos. Esse mesmo cenário também tem se apresentado em relação ao sexo dos adolescentes quanto à atividade física dos amigos ${ }^{15}$, ${ }^{17}$. Em relaçáo ao efeito moderador da idade e da condição socioeconômica na associação entre a prática de atividade física dos pais e dos amigos e a dos adolescentes são necessários mais estudos ${ }^{15,17}$.

Identificar e compreender quais fatores sociodemográficos moderam as associaçóes entre a prática de atividade física dos pais e dos amigos e a dos adolescentes ajudará a esclarecer em quais subgrupos a prática de atividade física dos pais e dos amigos podem exercer maior influência. Essas informaçóes podem ajudar a orientar as intervenções sobre atividade física em relação ao envolvimento dos pais e dos amigos conforme as características sociodemográficos dos adolescentes. O objetivo deste estudo foi analisar se fatores sociodemográficos moderam a associação da prática de atividade física dos pais e dos amigos com o nível de atividade física dos adolescentes.

[públicas e privadas], tamanho [número de alunos matriculados] e regióes do município [norte, sul, leste, oeste]; seguida da seleção aleatória de 135 turmas, distribuídas proporcionalmente por turno escolar [diurno e noturno] e série do ensino médio $\left[1^{\mathrm{a}}, 2^{\mathrm{a}}\right.$ e $3^{\mathrm{a}}$ séries $\left.]\right)$.

A coleta de dados ocorreu no período de maio a setembro de 2009 e foi realizada por uma equipe de seis estudantes de graduação em Educação Física, treinados e submetidos a um estudo piloto sob as mesmas condições do estudo principal. Todas as informaçóes foram coletadas por meio de questionário, previamente testado, preenchido pelos próprios escolares, em sala de aula, seguindo instruçóes prévias fornecidas pela equipe de coleta durante todo o processo para esclarecer dúvidas e orientar a forma correta de preenchimento. Para reduzir as perdas, o questionário foi aplicado de terça a quinta-feira, no segundo horário de aula.

As variáveis sociodemográficas analisadas neste 
estudo foram: sexo (masculino e feminino); idade em anos completos (categorizada em "14-15" e "1619 anos de idade"); classe econômica (metodologia da Associação Brasileira das Empresas de Pesquisa - ABEP)18, que leva em consideração a presença de bens materiais e de empregado mensalista na residência, e a escolaridade dos pais. A partir desses indicadores, as pessoas são agrupas nas classes econômicas A1, A2, B1, B2, C1, C2, D e E. Para fins de análise, elas foram reagrupadas em classes A/B (alta) e C/D/E (média-baixa).

$\mathrm{O}$ estado nutricional foi mensurado pelo índice de massa corporal $(\mathrm{IMC}=$ massa corporal $[\mathrm{kg}] /$ estatura $[\mathrm{m}]^{2}$ ), baseado nas medidas autorreferidas de massa corporal $(\mathrm{kg})$ e estatura $(\mathrm{cm})$. Os adolescentes foram categorizados em "sem excesso de peso corporal" (baixo peso + peso normal) e "com excesso de peso corporal" (sobrepeso + obesidade), conforme os pontos de corte sugeridos por Cole et $\mathrm{al}^{19}$.

A prática de atividade física do pai, da mãe e dos amigos foi mensurada pela seguinte questão: "Durante uma semana típica ou normal, em quantos dias... (sua mãe / seu pai / seus amigos), pratica $(m)$ atividades físicas como, por exemplo, caminhar, correr, andar de bicicleta, ir à academia de ginástica, fazer esportes ou musculação?”. As opçóes de respostas variavam de "nenhum" a "cinco ou mais dias por semana" e foram categorizadas em "nenhum dia", "1 a 2 dias" ou " 3 ou mais dias”. Essas questões tiveram os seguintes níveis de reprodutibilidade teste-reteste: prática de atividade física do pai - CCI = 0,92 (IC95\%: 0,90-0,94), da mãe - CCI = 0,90 (IC95\%: 0,87-0,92) e dos amigos - CCI = 0,82 (IC 95\%: 0,76-0,86).

A medida de atividade física dos adolescentes foi efetuada por questionário ${ }^{20}$. Os adolescentes informaram a frequência (dias/semana) e a duração (minutos/dia) das atividades físicas de intensidade moderada a vigorosa e o deslocamento ativo, praticados por pelo menos 10 minutos, na semana que antecedeu a coleta de dados, tomando como base uma lista com 24 atividades, com possibilidade de os adolescentes acrescentarem até duas atividades físicas. $\mathrm{O}$ nível de atividade física foi determinado pelo somatório dos produtos do tempo despendido nessas atividades e pelas suas respectivas frequências de prática. Os adolescentes com prática de atividade física igual ou superior a 300 minutos por semana foram considerados como "fisicamente ativos", e os demais como "fisicamente inativos".

Os dados foram digitados no programa EpiData 3.1 , seguindo um processo de dupla digitação, com checagem automática de consistência e amplitude dos valores de cada variável. A ferramenta "validar dupla digitação" deste programa foi utilizada para identificar possíveis erros de digitação. Todos os erros foram identificados e corrigidos com base nos valores registrados nos respectivos questionários.

Para a análise descritiva dos dados foi observada a distribuição por frequências, média, desvio padrão e intervalo de confiança de 95\%. O teste de Quiquadrado para tendência linear foi utilizado para comparar a proporção de adolescentes classificados como fisicamente ativos de acordo com a prática de atividade física dos pais e dos amigos.

A regressão logística binária foi utilizada para verificar a associação entre a prática de atividade física da mãe, do pai e dos amigos (variáveis independentes) e o nível de atividade física dos adolescentes (variável dependente). As variáveis independentes foram introduzidas no mesmo nível de análise e mantidas no modelo múltiplo (análise ajustada). Para avaliar se os fatores sociodemográficos (sexo, idade e classe econômica) moderavam estas associações, foram introduzidos termos de interação (multiplicação da possível variável moderadora com a variável independente - por exemplo: sexo*atividade física da mãe) no modelo. Termo de interação com valor $\mathrm{p}<0,05$ foi considerado como significativo, indicando a presença de moderação da variável sociodemográfica em análise. Nestes casos, foram realizadas análises estratificadas por cada variável sociodemográfica classificada como moderadora. Isso permitiu avaliar o sentido e a magnitude da medida de associação entre o nível de atividade física dos adolescentes e a prática de atividade física dos pais e dos amigos nos estratos dessas variáveis. As análises estatísticas foram realizadas no Stata 11.0, com nível de significância adotado menor que $5 \%$.

O estudo foi aprovado pelo Comitê de Ética em Pesquisa com Seres Humanos do Centro de Ciências da Saúde da Universidade Federal da Paraíba - Protocolo: 0062/2009. Todos os adolescentes menores de 18 anos de idade que participaram do estudo receberam autorização dos pais ou responsáveis e aqueles com mais de 18 anos de idade assinaram o termo de consentimento livre e esclarecido. 


\section{Resultados}

A amostra foi composta por 2.859 adolescentes de 14 a 19 anos de idade (perdas e recusas totalizaram 17,8\% dos casos), com média de idade de 16 anos (DP $=1,2$ ). Cálculos realizados a posteriori demonstraram que essa amostra tinha poder de $80 \%$ para detectar como significativas razões de odds iguais ou superiores a 1,40. A maioria dos adolescentes era do sexo feminino $(57,8 \%)$, tinha entre 16 e 19 anos de idade $(60,8 \%)$ e pertencia as classes econômicas média-baixa $(54,1 \%)$. A proporção de adolescentes fisicamente ativos foi igual a 50,2\% (IC95\%: 47,3-53,1), sendo estatisticamente mais elevada no sexo masculino (66,3\%; IC95\%: 63,7-69,0) comparado ao feminino (38,4\%; IC95\%: $36,1-40,8)$. A maioria dos pais (50,6\%; IC95\%: 48,5$52,2)$ e amigos (88,1\%; IC95\%: 87,0-89,4) praticava atividades físicas em um ou mais dias por semana, e 49,4\% (IC95\%: 47,6-51,2) das mães apresentaram essa frequência de prática (TABELA 1).

$\mathrm{Na}$ análise multivariável, observou-se que a prática de atividade física de ambos os pais e dos amigos se associou de forma positiva e significativa com o nível de atividade física dos adolescentes, indicando que quanto maior a prática de atividade física desses grupos maiores as chances de os adolescentes serem fisicamente ativos. A magnitude da medida de associação foi significativamente maior (sem intercessáo dos valores de IC95\%) para a frequência de prática de atividade física dos amigos de 3 ou mais dias por semana $(\mathrm{OR}=$ 3,01, IC95\%: 2,23-4,06) em relaçẫo à prática da mãe $(\mathrm{OR}=1,71, \mathrm{IC} 95 \%: 1,40-2,09)$ e do pai $(\mathrm{OR}=1,36$, IC95\%: 1,33-2,00) - TABELA 2.

Foram identificadas interaçóes significativas entre a prática de atividade física dos amigos e as variáveis sexo $(\mathrm{p}<0,001)$ e classe econômica $(\mathrm{p}=0,039)$, e entre a prática das mães e a classe econômica da família ( $\mathrm{p}$ $=0,035)$. Náo houve interaçáo significativa entre a variável idade e a prática de atividade física dos pais e dos amigos $(\mathrm{p}>0,005)$ - TABELA 2.

Na FIGURA 1 foram apresentados os resultados das análises de prática de atividade física dos amigos e das mães e o nível de atividade física dos adolescentes estratificadas por sexo e classe econômica. Os resultados dessas análises demonstraram que a magnitude da medida de associação entre a prática de atividade física dos amigos e o nível de atividade física dos adolescentes do sexo masculino foi mais que o dobro $(\mathrm{OR}=5,02$, IC95\%: 2,73-9,26) quando comparada a do sexo feminino $(\mathrm{OR}=2,21$, IC95\%: 1,55-3,14) - Figura 1A. Já a magnitude da medida de associação entre a prática de atividade física dos amigos e o nível de atividade física dos adolescentes de classe econômica alta foi maior $(\mathrm{OR}=3,90$, IC95\%: 2,39-6,39) do que a identificada na classe econômica média-baixa $(\mathrm{OR}=2,27$, IC95\%: 1,53-3,37) - FIGURA 1B. Nos adolescentes de classe econômica alta foi identificado aumento de até $80 \%$ na chance ser fisicamente ativo quando a mãe também era fisicamente ativa 3 ou mais dias por semana (FIGURA 1C).

Verificou-se uma tendência linear de aumento na prevalência de nível suficiente de atividade física dos adolescentes conforme a prática de atividade física do pai, da máe e dos amigos (TABELA 2), sendo de maior magnitude de prevalência (sem intercessáo dos valores de IC95\%) observada nos adolescentes do sexo masculino para a prática dos amigos (1-2 dias/ sem. $=52,6 \%$; IC95\%: 47,3-57,9; 3 ou mais dias/ sem. $=75,3 \%$; IC95\%: 72,2-78,2) em relação ao sexo feminino (1-2 dias/sem. = 35,4\%; IC95\%: 31,8-39,3; 3 ou mais dias/sem. = 46,2\%; IC95\%: 42,6-49,8) e nos de classe econômica $\mathrm{A} / \mathrm{B}$ quando a prática dos amigos foi frequente em 3 ou mais dias por semana (62,9\%; IC95\%: 57,4-68,0) comparado aos de classe C/D/E (52,3\%; IC95\%: 47,3-57,3) - resultados não disponíveis em tabela. 
TABELA 1 - Características da amostra de adolescentes do município J oão Pessoa (PB), 2009.

\begin{tabular}{|c|c|c|}
\hline Variável & $\mathbf{n}$ & $\%$ \\
\hline \multicolumn{3}{|l|}{ Sexo } \\
\hline Masculino & 1.206 & 42,2 \\
\hline Feminino & 1.653 & 57,8 \\
\hline \multicolumn{3}{|l|}{ Faixa etária } \\
\hline $14-15$ anos & 1.128 & 39,2 \\
\hline 16-19 anos & 1.746 & 60,8 \\
\hline \multicolumn{3}{|l|}{ Cor da pele } \\
\hline Branco & 929 & 32,6 \\
\hline Não Branco & 1.915 & 67,4 \\
\hline \multicolumn{3}{|l|}{ Trabalho } \\
\hline Trabalha & 366 & 13,1 \\
\hline Não trabalha & 2.436 & 86,9 \\
\hline \multicolumn{3}{|l|}{ Classe econômica } \\
\hline A/B (alta) & 1.161 & 45,8 \\
\hline C/D/E (média-baixa) & 1.372 & 54,2 \\
\hline \multicolumn{3}{|l|}{ Escolaridade do pai } \\
\hline Fundamental & 1.391 & 50,0 \\
\hline Médio completo & 895 & 32,1 \\
\hline Superior completo & 497 & 17,9 \\
\hline \multicolumn{3}{|l|}{ Escolaridade da mãe } \\
\hline Fundamental & 1.391 & 48,9 \\
\hline Médio completo & 906 & 31,8 \\
\hline Superior completo & 550 & 19,3 \\
\hline \multicolumn{3}{|c|}{ Prática de atividade física da mãe } \\
\hline $0 \mathrm{dia} /$ semana & 1.435 & 50,6 \\
\hline 1-2 dias/semana & 611 & 21,6 \\
\hline 3 ou mais dias/semana & 786 & 27,8 \\
\hline \multicolumn{3}{|c|}{ Prática de atividade física do pai } \\
\hline $0 \mathrm{dia} / \mathrm{semana}$ & 1.374 & 49,6 \\
\hline $1-2$ dias/semana & 659 & 23,8 \\
\hline 3 ou mais dias/semana & 734 & 26,6 \\
\hline \multicolumn{3}{|c|}{ Prática de atividade física dos amigos } \\
\hline $0 \mathrm{dia} / \mathrm{semana}$ & 333 & 11,8 \\
\hline 1-2 dias/semana & 965 & 34,3 \\
\hline 3 ou mais dias/semana & 1.518 & 53,9 \\
\hline \multicolumn{3}{|c|}{ Atividade física dos adolescentes } \\
\hline Fisicamente inativo & 1.430 & 49,8 \\
\hline Fisicamente ativo & 1.444 & 50,2 \\
\hline
\end{tabular}


Mendonça G, et al.

TABELA 2 - Análise de regressão logística binária bruta e ajustada para associação entre prática de atividade física dos pais e dos amigos, e o nível de atividade física dos adolescentes de J oão Pessoa (PB), 2009.

*Ajustada por sexo, faixa etária, classe econômica e índice de massa corporal; IC95\%: Intervalo de confiança de $95 \%$. Negrito $=$ diferença significativa $(p<0,05)$ para o termo de interação.

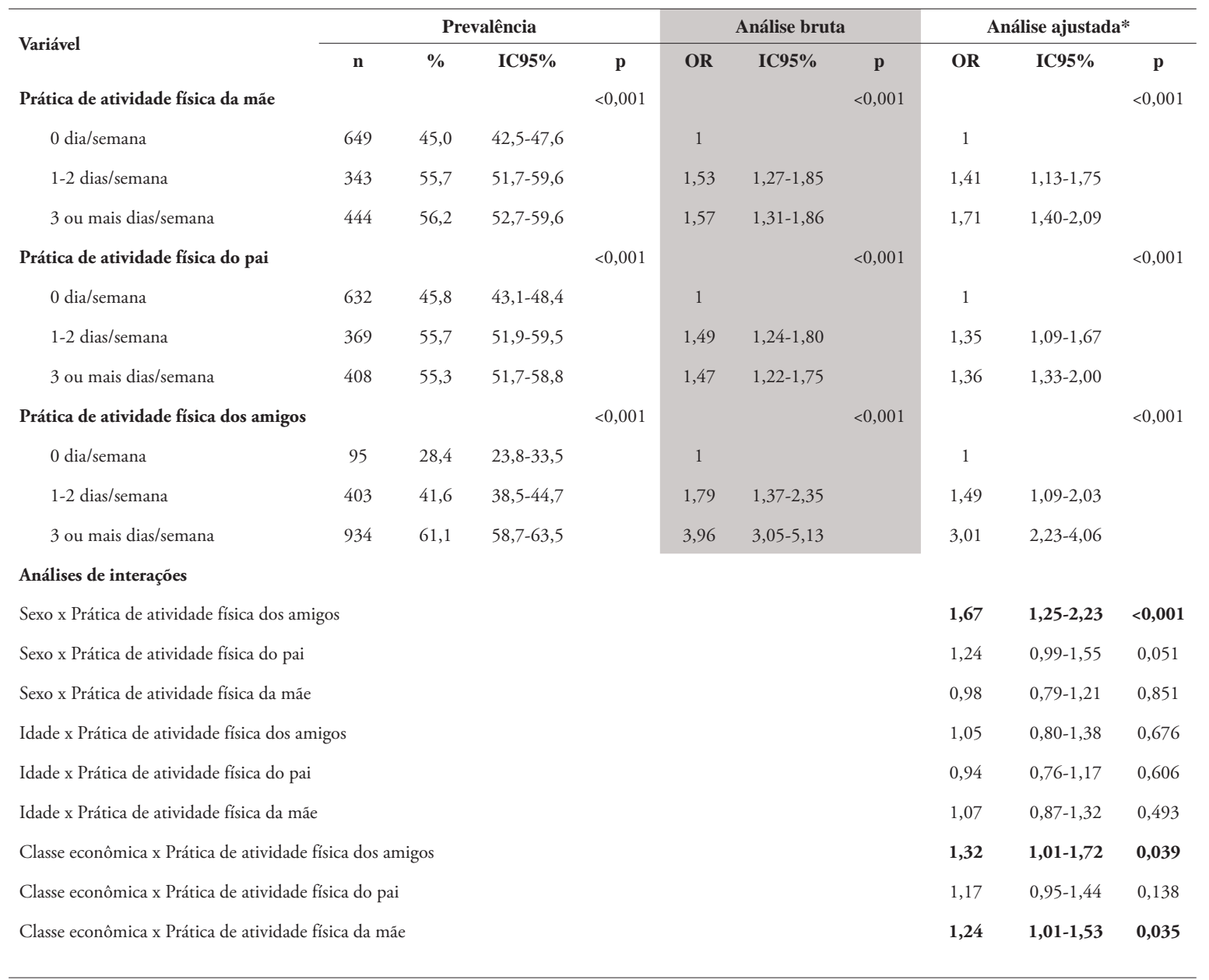


FIGURA 1 - Análise de regressão logística binária ajustada* para associação entre prática de atividade física dos pais e dos amigos e o nível de atividade física dos adolescentes de J oão Pessoa (PB), 2009.

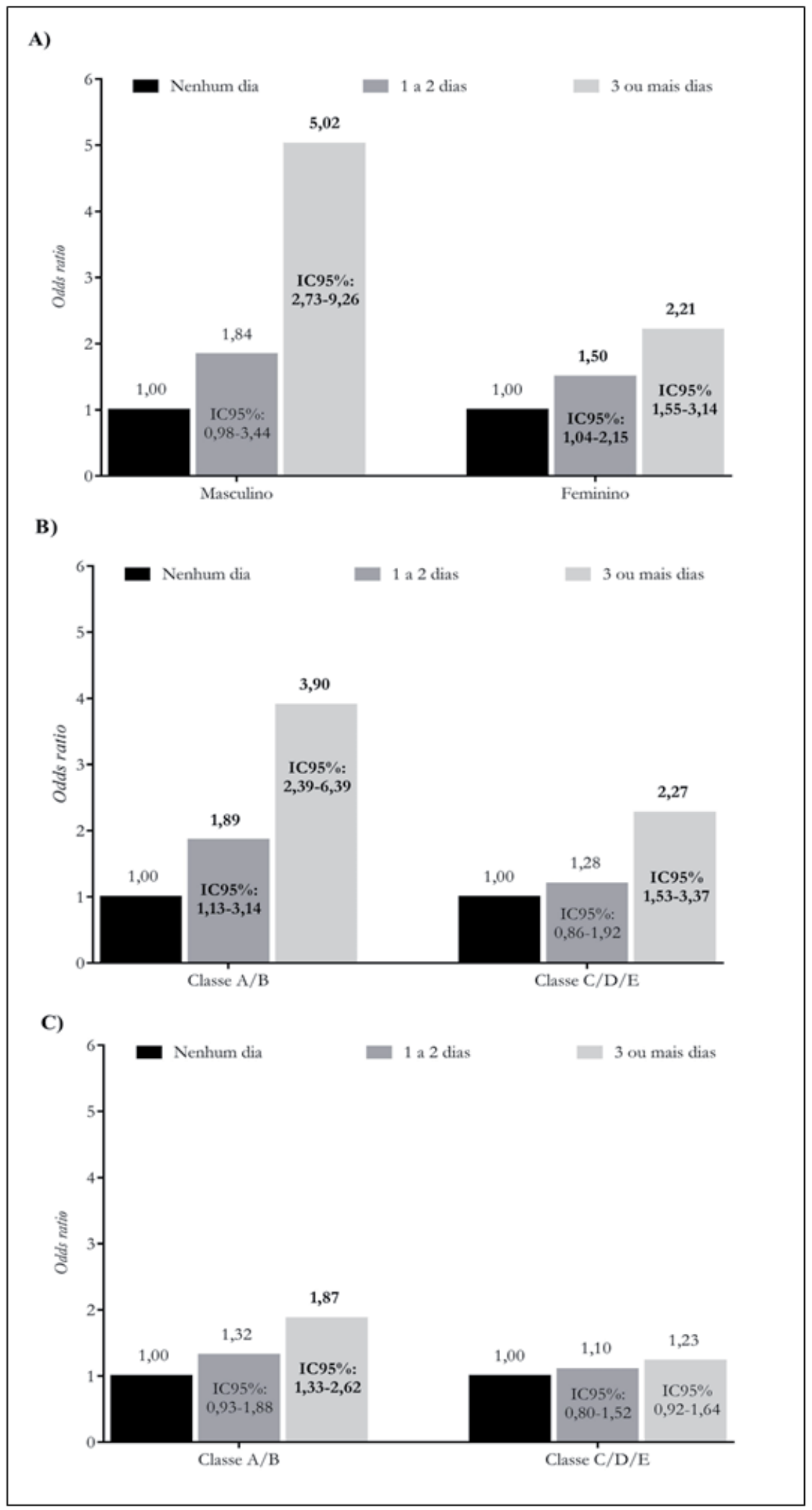

A) Odds ratio

da associação

entre prática dos

amigos e nível de

atividade física

dos adolescentes

estratificado por sexo

B) Odds ratio

da associação

entre prática dos

amigos e nível de

atividade física

dos adolescentes

estratificado por

classe econômica;

C) Odds ratio

da associação

entre prática das

mães e nível de

atividade física

dos adolescentes

estratificado por

classe econômica.

IC95\%: Intervalo de

confiança de $95 \%$.

*Ajustada por sexo

faixa etária, classe

econômica e índice

de massa corporal;

Negrito = associação

significativa (valor $p<$ $0,005)$. 


\section{Discussão}

O principal objetivo deste estudo foi analisar se os fatores sociodemográficos moderam a associação da prática de atividade física dos pais e dos amigos com o nível de atividade física dos adolescentes. De forma geral, foi identificado que maior prática de atividade física dos pais e dos amigos estava associada com maiores níveis de atividade física dos adolescentes. No entanto, a magnitude das medidas de associação entre a prática dos amigos e o nível de atividade física dos adolescentes foi maior para os do sexo masculino e de classe econômica alta em relação a seus pares. Também foi identificado que a prática da mãe se associou com o nível de atividade física nos adolescentes de classe econômica alta. Esses resultados, em conjunto, indicam que os fatores sociodemográficos moderam a associação entre essas variáveis.

No presente estudo, adolescentes que percebiam seus pais e, sobretudo, os seus amigos participando de atividades físicas com maior frequência, apresentaram maiores chances de serem fisicamente mais ativos. Esses resultados são semelhantes aos de outros estudos ${ }^{11-13}$. A modelagem do comportamento está entre as principais formas de influência social exercida pelos principais grupos sociais de convívio dos adolescentes, sendo frequentemente citada como um importante fator que influência a atividade física desse grupo ${ }^{6,13}, 14$. A modelação por meio da observação direta e o convívio dos adolescentes com os pais e os amigos são aspectos que podem determinar a adesão de comportamentos semelhantes ${ }^{8}$, dentre eles a prática de atividade física.

Como observado em estudos com adolescentes de diferentes países ${ }^{7,10,13}$, no presente estudo, verificou-se uma maior magnitude de associação entre nível de atividade física dos adolescentes e a prática de atividade física dos amigos do que a prática dos pais (pai e mãe). É possível que o efeito da modelação do comportamento para a prática de atividade física seja maior nos amigos, uma vez que com passar dos anos, os adolescentes tendem a passar mais tempo na companhia dos amigos do que dos pais ${ }^{11}$. Sendo assim, passariam a ser mais influenciados pelas tendências, hábitos e normas partilhadas com os amigos ${ }^{13,21}$. Outra explicação pode está atrelada a possibilidade de que os adolescentes recordariam com maior precisão da participação dos amigos em atividades físicas por serem essas práticas realizadas, em sua maioria, de forma conjunta. Normalmente, os adolescentes partilham das mesmas atividades que seus amigos, frequentam ambientes de prática semelhantes, dividem os gostos pelas mesmas atividades e adotam comportamentos parecidos de prática para pertencer ao seu grupo social ${ }^{13}$. Em estudo de revisão sistemática, SAWKA, et al. ${ }^{21}$ identificaram que os adolescentes podem adotar um comportamento em decorrência da influência social exercida pelos amigos ou por meio da seleçáo de amigos que apresentassem alguns interesses em comum. No que se refere a atividade física, a escolha das amizades estaria relacionada a aproximação dos adolescentes igualmente ativos fisicamente ${ }^{21}$.

Apesar de a prática de atividade física dos amigos ter se mostrado associada a maiores níveis de atividade física em adolescentes de ambos os sexo, a magnitude da associação foi significativamente maior no sexo masculino comparado ao feminino. Esse achado indica o efeito moderador dessa variável para essa associaçáo, aspecto que também foi observado em outros estudos com adolescentes ${ }^{14,15,22}$. A maior participaçáo dos adolescentes do sexo masculino em atividades físicas coletivas ${ }^{23,24}$, o fato de receberem mais apoio social para a prática de atividade física dos ciclos de amizades ${ }^{25,26}$ e estar mais envolvido em atividades realizadas ao ar livre ${ }^{27}$, são aspectos que aumentam a possibilidade de novas amizades e consecutivamente uma maior potencial de modelação de suas práticas de atividade físicas.

É importante destacar também que os adolescentes do sexo masculino praticam e valorizem mais a participaçáo em esportes, por exemplo, como futebol e voleibol ${ }^{23}$. Sendo assim, é possível que eles se espelhem nos seus ídolos dessas modalidades. Por outro lado, os adolescentes do sexo feminino apresentam menor prática de atividade física, principalmente em esportes ${ }^{1,2,28}$. Essas atividades poderiam facilitar o conhecimento de novos amigos em quem as adolescentes pudessem se espelhar em suas práticas ${ }^{23,24}$. Outro aspecto que precisa ser considerado é o fato de que as adolescentes são estimuladas desde cedo para se envolverem em atividades domésticas e seguirem "regras sociais" ou "estigmas" que as induzem a permanecer mais 
tempo dentro de casa ${ }^{15,27}$. Isso ajudaria a diminuir as oportunidades de maior envolvimento com outros adolescentes a quem pudessem se espelhar para maiores práticas.

O presente estudo identificou que a classe econômica moderou a associação entre a prática de atividade física dos amigos e o nível de atividade física dos adolescentes. Apesar de estas variáveis estarem positivamente associadas nos adolescentes de classes econômicas alta e média-baixa, a magnitude de associação foi maior nos de classe econômica alta. Adolescentes de condição econômica alta apresentam maiores oportunidades de encontrar amigos e realizar suas atividades físicas nos períodos de lazer, principalmente em atividades estruturadas e/ou coletivas, diferentemente daqueles de menor condição socioeconômica ${ }^{29}$. Este argumento pode ajudar a explicar esses resultados, tendo em vista que para que essas práticas aconteçam há a necessidade de locais específicos (por exemplo: quadras, ginásios esportivos, academias e clubes recreativos) ${ }^{15}$. Esses locais seriam mais acessíveis para as pessoas de classe socioeconômica mais alta ${ }^{29}$ e possibilitariam maiores vínculos de amizade, consequentemente, os adolescentes poderiam se espelhar nas práticas de atividade física desses amigos.

Outro achado importante deste estudo foi identificar a associação entre maior prática de atividade física das mães e a maiores níveis de atividade física nos adolescentes de classe econômica alta, caracterizando o efeito moderador desta variável. Este resultado deve-se ao fato de que as máes de melhor condição socioeconômica, geralmente, residem em bairros com melhor infraestrutura para a prática de atividades físicas (presença de parques, praças, pista de corrida/ caminhada, ciclovias ou ciclofaixas) ${ }^{22}$, possuem maior grau de conhecimento sobre a adoção de comportamentos positivos à saúde, tendo assim maiores chances de se tornarem mais fisicamente ativas. Sendo assim, elas passariam a ser exemplos ou referência para seus filhos (adolescentes) em termos de modelo para a prática de atividade física ${ }^{30}$.

É possível que entre as mães de condição econômica alta a prática de atividade física seja mais valorizada, e estas por sua vez podem transmitir maior apoio social para as práticas de seus filhos, por meio de palavras de encorajamento, fornecimento de transporte, praticar em conjunto ou assistir a prática deles em atividades físicas $^{25}$. Já as mães de menor condição socioeconômica são frequentemente menos ativas fisicamente ${ }^{1,2}$, apresentam menor nível de conhecimento sobre a importância da atividade física para a saúde, fornecem menos apoio social para a atividade física dos filhos ${ }^{25}$, servindo possivelmente como modelos negativos para a prática deles. Mães de menor nível socioeconômico exigem maior envolvimento dos seus filhos em compromissos sociais como trabalhar, cuidar da casa, realizar cursos e estágios para capacitação ${ }^{12}$, resultando em menor nível de prática de atividade física por partes destes e também não favorecendo o processo de modelagem da prática da mãe para o filho.

Os pontos fortes deste estudo incluem: a utilização de uma amostra representativa de adolescentes do ensino médio de escolas públicas e privadas; o instrumento utilizado na coleta de dados foi previamente validado e a coleta de dados foi realizada por equipe treinada. Entretanto, o presente estudo apresenta algumas limitaçóes: trata-se de um estudo transversal, não sendo possível estabelecer uma relação causal entre a atividade física dos pais e dos amigos e a atividade física dos adolescentes; a medida da atividade física dos pais e dos amigos foi baseada na percepção do adolescente e náo considerou o tempo de prática por dia. Esse aspecto pode ter superestimado a prática de atividade física dos pais e dos amigos quando essas práticas foram relatadas pelos adolescentes mais ativos e, consecutivamente, superestimou as magnitudes das medidas de associação entre as variáveis em estudo.

Conclui-se que a associação entre a prática de atividade física dos amigos e das mães e o nível de atividade física dos adolescentes variou conforme o sexo e a classe econômica da família, demonstrando que os fatores sociodemográficos moderam a associação entre essas variáveis. Nos adolescentes do sexo masculino e de classe econômica alta foram identificadas maiores magnitudes nas medidas de associação entre a prática de atividade física dos amigos e a dos adolescentes. A prática de atividade física da mãe se associou apenas ao nível de atividade física dos adolescentes de classe econômica alta.

O desenvolvimento de intervençóes para promoção da atividade física em adolescentes deve investir em açóes que possibilitem um maior envolvimento dos pais e dos amigos com prática de atividade física para que estes possam influenciar por meio da modelação do comportamento a prática de atividade física dos adolescentes. No entanto, as ações propostas devem considerar as caraterísticas sociodemográficas dos adolescentes, tendo em vista que a prática de atividade física dos amigos e das mães foram mais importantes para influenciar o nível de atividade física dos adolescentes do sexo masculino e os de classe econômica mais alta. 


\title{
Agradecimentos
}

À Coordenação de Aperfeiçoamento de Pessoal de Nível Superior (CAPES) pela bolsa de estudos e pesquisa. À Secretaria de Educação da Paraíba, aos gestores, professores e alunos das escolas que participaram do estudo. Aos estagiários do Curso de Educaçáo Física que fizeram parte da equipe de coleta de dados. Ao Programa de Iniciação Científica e Tecnológica - PIBIC da Universidade Federal da Paraíba.

\section{Conflitos de interesse}

Os autores declaram não haver conflitos de interesse.

\begin{abstract}
Do socio-demographic factors moderate association between practice of physical activity of parents and friends with level of physical activity of adolescents?

The objective of this study was to analyze if the sociodemographic factors moderate the association of physical activity practice of parents and friends, and the level of physical activity among adolescents. It was an epidemiological study with 2,859 adolescents (57.8\% female) $14-19$ years old of the city of Joao Pessoa (PB). The level of physical activity among adolescents and the physical activity of parents and friends were measured by questionnaires. The prevalence of physically active adolescents was 50.2\% (95\% Cl: 47.3-53.1), being higher in males (66.3\%; 95\% Cl: 63.7-69.0) compared to female (38.4\%; 95\% Cl: 36.1-40.8). The results of multivariate analysis showed that there is an association between physical activity of parents and friends and the level of physical activity among adolescents. However, this association is moderated by sociodemographic factors (sex and economic class). The magnitude of the measure of the association between the practice of physical activity of friends and adolescents was higher in male and high economic class adolescents. The physical activity of the mother was associated significantly and positively with the level of physical activity in adolescents of high economic class. We conclude that sociodemographic factors moderate the association of physical activity from friends and from the mother with the level of physical activity of adolescents. The magnitude of the association between these variables varied with sex and economic class of adolescents.
\end{abstract}

KEYWORDS: Motor activity; Adolescents; Parents; Friends.

\section{Referências}

1. Hallal P, Andersen LB, Bull FC, Guthold R, Haskell W, Ekelund U. Global physical activity levels: surveillance progress, pitfalls, and prospects. Lancet. 2012; Series 247-257.

2. Townsend N, Wickramasinghe K, Williams J, Bhatnagar P, Rayner M. Physical Activity Statistics 2015. London, UK: British Heart Foundation; 2015.

3. Bauman AE, Reis RS, Sallis JF, Wells JC, Loos RJ, Martin BW. Correlates of physical activity: why are some people physically active and others not? Lancet. 2012; Series 258-271.

4. Craggs C, Corder K, van Sluijs EMF, Griffin SJ. Determinants of change in physical activity in children and adolescents: a systematic review. Am J Prev Med. 2011;40:645-658.

5. Chung SJ, Ersig AL, McCarthy AM. The Influence of peers on diet and exercise among adolescents: a systematic review. J Pediatr Nurs. 2017;36:44-56.

6. Wilson DK, Van Horn ML, Kitzman-Ulrich H, Saunders R, Pate R, Lawman HG, Hutto B, Griffin S, Zarrett N, Addy CL, Mansard L, Mixon G, Brown PV. Results of the "Active by Choice Today" (ACT) randomized trial 
for increasing physical activity in low-income and minority adolescents. Health Psychol. 2011;30:463-471.

7. Wilson AN, Dollman J. Social influences on physical activity in Anglo-and Vietnamese-Australian adolescent males in a single sex school. J Sci Med Sport. 2007;10:147-155.

8. Bandura A. Social cognitive theory: An agentic perspective. Annu Rev Psychol. 2001;52:1-26.

9. Bandura A. Self-efficacy: Toward a unifying theory of behavioral change. New York, NY, US: Psychology Press; 1999.

10. Ornelas IJ, Perreira KM, Ayala GX. Parental influences on adolescent physical activity: a longitudinal study. Int J Behav Nutr Phys Act. 2007;4:3.

11. Kirby J, Levin KA, Inchley J. Parental and peer influences on physical activity among Scottish adolescents: a longitudinal study. J Phys Act Health. 2011;8:785.

12. Lemos Nd, Nakamura PM, Grisi RNdF, Kokubun E. Association between the parent's leisure physical activity level and their children`s physical activity level. Rev Bras Ativ Fis Saude. 2012;15:95-100.

13. Fitzgerald A, Fitzgerald N, Aherne C. Do peers matter? A review of peer and/or friends' influence on physical activity among American adolescents. J Adolesc. 2012;35:941-958.

14. Trost SG, Loprinzi PD. Parental influences on physical activity behavior in children and adolescents: a brief review. Am J Lifestyle Med. 2011;5:171-181.

15. Seabra AF, Mendonça DM, Thomis MA, Anjos LA, Maia JA. Biological and socio-cultural determinants of physical activity in adolescents. Cad Saude Publica. 2008;24:721-736.

16. Bauman AE, Sallis JF, Dzewaltowski DA, Owen N. Toward a better understanding of the influences on physical activity: the role of determinants, correlates, causal variables, mediators, moderators, and confounders. Am J Prev Med. 2002;23:5-14.

17. Yao CA, Rhodes RE. Parental correlates in child and adolescent physical activity: a meta-analysis. Int J Behav Nutr Phys Act. 2015;12:10.

18. Brazil Economic Classification Criterion - [database on the Internet]2009 [cited November 26th, 2009]. Available from: http://www.abep.org/new/codigosCondutas.aspx.

19. Cole TJ, Bellizzi MC, Flegal KM, Dietz WH. Establishing a standard definition for child overweight and obesity worldwide: international survey. BMJ. 2000;320:1240-1243.

20. Farias Júnior JC, Lopes AS, Mota J, Santos MP, Ribeiro JC, Hallal PC. Validity and reproducibility of a physical activity questionnaire for adolescents: adapting the Self-Administered Physical Activity Checklist. Rev Bras Epidemiol. 2012;15:198-210.

21. Sawka KJ, McCormack GR, Nettel-Aguirre A, Hawe P, Doyle-Baker PK. Friendship networks and physical activity and sedentary behavior among youth: a systematized. Int J Behav Nutr Phys Act. 2013;10.

22. Farias Júnior JC, Lopes AS, Mota J, Hallal PC. Physical activity practice and associated factors in adolescents in Northeastern Brazil. Rev Saude Publica. 2012;46:505-515.

23. Azevedo Junior MR, Araújo CLP, Pereira FM. Physical and sports activities in adolescence: changes in choices over the last decades. Rev Bras Educ Fis Esp. 2006;20:51-58.

24. Silva SM, Knuth AG, Duca GFD, Camargo MBJ, Cruz SH, Castagno V, Menezes AMB, Hallal PC. Individual and collective sports practice and associated factors among adolescents belonging to a birth cohort study. Rev Bras Educ Fis Esp. 2009;23:263-274.

25. Mendonça G, Cheng LA, Melo EN, Farias Junior JC. Physical activity and social support in adolescents: a systematic review. Health Educ Res. 2014;30:822-839.

26. Mendonça G, Farias Júnior JC. Physical activity and social support in adolescents: analysis of different types and sources of social support. J Sports Sci. 2015;33:1942-1951.

27. Gonçalves H, Hallal PC, Amorim TC, Araújo CLP, Menezes A. Sociocultural factors and physical activity level in early adolescence. Rev Panam Salud Pública. 2007;22:246-253.

28. Mendonça G, Cheng LA, Farias Júnior JC. Padrôes de prática de atividade física em adolescentes de um município da região Nordeste do Brasil. Ciênc Saúde Coletiva. 2016;[Citado em 20/12/2017]. Está disponível em: http:// www.cienciaesaudecoletiva.com.br/artigos/padroes-de-pratica-de-atividade-fisica-em-adolescentes-deum-municipioda-regiao-nordeste-do-brasil/15921?id=15921.

29. Bengoechea EG, Sabiston CM, Ahmed R, Farnoush M. Exploring links to unorganized and organized physical activity during adolescence: the role of gender, socioeconomic status, weight status, and enjoyment of physical education. Res Q Exerc Sport. 2010;81:7-16. 
Mendonça G, et al.

30. Hanson MD, Chen E. Socioeconomic status and health behaviors in adolescence: a review of the literature. J Behav Med. 2007;30:263-285.

ENDEREÇO

Gerfeson Mendonça Universidade Federal da Paraíba Centro de Ciência da Saúde Departamento de Educação Física Campus I - Cidade Universitária, Submetido: 14/01/2016 Revisado: 11/01/2018 Aceito: 09/04/2018 